\title{
Calidad de vida en el paciente pediátrico con Enfermedad Renal Crónica
}

\author{
Pablo Jesús López Soto
}

Diplomado Universitario de Enfermería. Universidad de Córdoba

\section{Resumen}

Objetivos: Conocer la producción científica en los últimos seis años sobre la calidad de vida de los pacientes pediátricos con enfermedad renal crónica.

Métodos: Las bases de datos consultadas fueron PubMed, Scopus, Science Direct, ProQuest, Web of Knowledge y SciVerse. Sólo se incluyeron los artículos científicos escritos en inglés o español y que tuvieran un diseño de corte transversal. Las principales variables que afectan a la calidad de vida eran analizadas, entre las que destacaba la modalidad terapéutica. Además, se estudió los distintos cuestionarios de medición de la calidad de vida, así como las diferencias en la percepción entre los pacientes y sus familias.

Resultados: Trece documentos fueron encontrados: sólo uno de ellos empleó un cuestionario específico para este tipo de pacientes, diez cuestionarios generales y los dos documentos que empleaban los dos tipos. Los cuestionarios generales estudiaban la variable de "modalidad terapéutica" que se emplea, así como otros factores que afectan a la calidad de vida tales como trastornos del sueño y mentales; por otro lado, el cuestionario específico sólo estudió la variable "modalidad terapéutica". En los dos estudios que emplean ambos tipos de cuestionarios la principal variable de estudio es la "modalidad terapéutica" y se permite una comparación entre los dos tipos de cuestionarios.

Correspondencia:

Pablo Jesús López Soto

Calle San Cayetano $\mathrm{n}^{\circ} 21$

04140. Carboneras. Almería

e-mail: pablolopezsoto90@gmail.com
La mayor parte de los estudios emplean un informe parental para determinar la calidad de vida del paciente pediátrico con enfermedad renal crónica.

Conclusiones: Los pacientes pediátricos con enfermedad renal crónica tienen una peor calidad de vida que los pacientes pediátricos con otras enfermedades crónicas tales como diabetes. Los niños trasplantados 0 en tratamiento conservador poseen una mejor calidad de vida que los sometidos a tratamiento sustitutivo.

\section{PALABRAS CLAVE:}

- CALIDAD DE VIDA

- NIÑO

- ENFERMEDAD RENAL CRÓNICA

- PACIENTE PEDIÁTRICO

\section{Quality of life in paediatric patients with Chronic Renal Disease}

\section{Abstract}

Aims: To look for the scientific literature in the last six years about the quality of life of pediatric patients with chronic kidney disease.

Methods: The next databases were used: PubMed, Scopus, Science Direct, ProQuest, Web of Knowledge and SciVerse. Only scientific papers written either in English or Spanish as well as having a crosssectional design were considered. The main variables affecting the quality of life, amongst which stand out therapeutics, were analyzed. Also, the different ways for measuring the quality of life as well as 
differences in its perception between patients and their families were studied.

Results: Thirteen papers were found: only one of them used specifically prepared questionnaire for this type of patients, ten used general questionnaires and the last two papers mixed both types. The general questionnaires studied the variable "therapeutic modality" used as well as other factors affecting the quality of life such as dream and mental disorders; on the other side, the specific questionnaire only studied the variable "therapeutic modality". The cases in which both types of questionnaires were used allowed a comparison between the two approaches, being "therapeutic modality" the main variable to be considered.

Most papers included a family report for evaluating the quality of life.

Conclusions: Pediatric patients with chronic kidney disease have a worse quality of life than people with other chronic diseases such as, for instant, diabetes. Children either transplanted or under a conservative treatment enjoyed a better quality of ife that those subjected to dialysis.

\section{KEY WORDS: \\ - QUALITY OF LIFE \\ - CHILD \\ - CHRONIC KIDNEY DISEASE \\ - PEDIATRICS}

\section{Introducción}

Se va a desarrollar una revisión descriptiva de toda la producción científica relacionada con la calidad de vida del paciente pediátrico (desde el nacimiento hasta los 18 años) con enfermedad renal crónica. El artículo se va a estructurar en: marco teórico, objetivo, metodología, resultados y conclusiones. Además se incluyen anexos, referencias bibliográficas y una propuesta de proyecto.

\section{Marco teórico}

Según las guías de práctica clínica de la K/D0QI de la National Kidney Foundation (NFK) en 2002 definió enfermedad renal crónica (ERC) como daño renal durante $>3$ años, caracterizado por anomalías funcionales o estructurales, con o sin disminución de la tasa de filtración glomerular (GFR) tal como se manifiesta ya sea por alteraciones patológicas o marcadores de daño renal (es decir, análisis de orina o estudio de imagen anormal) o una GFR por debajo de $60 \mathrm{ml} / \mathrm{min} / 1.73$ $\mathrm{m}^{2}$ durante dos o tres meses, con o sin evidencia de lesión renal ${ }^{1}$.

La enfermedad renal crónica (ERC) en la edad pediátrica (desde el nacimiento hasta los 18 años) es poco frecuente, pero cuando aparece, lo hace con efectos bastante importantes para el desarrollo del niño, desarrollando una alta morbilidad.

Según el Registro Español Pediátrico de Insuficiencia Renal (REPIR II) en 2010, la incidencia de la ERC no terminal era de 8,66 casos por millón de población en menores de 18 años y la prevalencia de $71,06^{2}$. En el caso de los pacientes pediátrico con ERCT, los datos epidemiológicos corren a cargo de la Asociación Española de Nefrología Pediátrica (AENP), que según los últimos datos obtenidos en el año 2010 hay 60 niños en programas de diálisis y trasplantes y el tiempo medio para el trasplante no suele durar el año, practicándose una media de entre 60 y 70 trasplantes anuales ${ }^{3}$. La enfermedad renal crónica es una patología de larga duración, progresiva e irreversible, en el niño esto se acentúa más al abarcar un gran período de su vida e incluso toda su vida. En este caso, no solo es el paciente el que se somete a la enfermedad y tratamiento, si no que la familia también lo está. La existencia de un niño enfermo crónico supone una alteración de la vida cotidiana, actividades y proyectos de otros miembros de la familia, relación entre padres o entre los hermanos sanos 4 .

La calidad de vida (CV) puede definirse como la percepción subjetiva de bienestar general que resulta de la evaluación que hace el individuo de diversos dominios o áreas de su vida 5 . Se trata de un constructo multidimensional, que incorpora aspectos tales como la salud física de la persona, su estado psicológico, grado de independencia, relaciones sociales, factores ambientales y creencias personales.

La prevalencia de enfermedades crónicas, en la mayoría de los países, para las cuales no existe una curación total y donde el objetivo del tratamiento 
es atenuar o eliminar síntomas, evitar complicaciones y mejorar el bienestar de los pacientes, lleva a que las medidas clásicas de resultados en medicina (mortalidad, morbilidad, expectativa de vida) no sean suficientes para evaluar la calidad de los servicios de salud'.

En este contexto, la medida de la Calidad de Vida Relacionada con la Salud (CVRS) incorpora la percepción del paciente, como una necesidad en la evaluación de resultados en salud, debiendo para ello desarrollar los instrumentos necesarios para que esa medida sea válida y confiable y aporte evidencia empírica con base científica al proceso de toma de decisiones en salud ${ }^{7}$.

Por tanto, se va a considerar CVRS a cómo el paciente percibe y reacciona frente a su estado de salud, a los aspectos de la vida que pueden ser atribuidos a la enfermedad y a su terapéutica. En general, a todos los aspectos de la vida de un individuo que están influidos por la salud². La CVRS ha sido uno de los conceptos que en el campo de la salud más se ha utilizado de manera indistinta al de calidad de vida, siendo muy pocos los autores que hacen una distinción con el término calidad de vida general ${ }^{8}$.

Ya que para algunos autores, la CVRS es frecuente $\neg$ mente identificada como una parte de la calidad de vida general, mientras que otros sugieren que su uso es a menudo intercambiable con el de CV ${ }^{9}$. Los avances médicos en los cuidados para niños con enfermedad renal crónica en las distintas modalidades terapéuticas han resultado en una relativa mejora a largo plazo en la supervivencia del paciente comparándolo con pacientes adultos con ERC, de lo que se deduce que habrá más pacientes pediátricos con ERC que van a llegar a edad adulta ${ }^{10}$.

Por tanto, los cuidados óptimos para el paciente pediátrico con ERC no sólo requieren atención del manejo médico sino también de la evaluación de los factores de CVRS que podría promover o prevenir una exitosa transición a la edad adulta. La calidad de vida en la infancia refleja el punto de vista del niño o de su familia acerca del impacto de la enfermedad en su vida cotidiana, abarcando diversos factores y no sólo los relacionados directamente con el estado 0 la gravedad de la enfermedad. La enfermedad renal crónica supone una crisis para la vida familiar como sistema y también, de forma particular, para cada uno de sus integrantes 4 . Si la "calidad de vida" es complicada de definir y de llegar a un acuerdo por lo que se entiende como tal, también es complicado medirla. Habitualmente la medida de la "calidad de vida" se realiza a partir del análisis de las respuestas de los individuos a un cuestionario estandarizado que comprende distintas dimensiones. Cada dimensión está constituida por distintas preguntas o ítems que se encentran categorizadas por escalas ${ }^{4,11}$.

Los cuestionarios pediátricos de la calidad de vida, al igual que en los de los adultos, se pueden clasificar en genéricos y específicos según las características de la población para la cual han sido creados.

Los instrumentos genéricos intentan dar una aproximación general del estado de salud, se utilizan en cualquier población de estudio independientemente de la enfermedad que afecta a dicha población y analizan contenidos comunes a cualquier situación ${ }^{12}$. Los instrumentos específicos, por su parte, están dirigidos a una enfermedad y sus dimensiones intentan profundizar en aspectos de la CVRS de un síntoma, proceso agudo o crónico, capturando con mayor precisión el impacto de los tratamientos y de las intervenciones sanitarias ${ }^{5,13}$.

\section{Objetivos}

El objetivo general de estudio es:

- Conocer la producción científica que hay sobre calidad de vida del paciente pediátrico con enfermedad renal crónica (PPERC) en un período de 6 años (desde 2007 hasta 2012).

Los objetivos específicos:

- Conocer niveles de la calidad de vida en PPERC en las distintas modalidades terapéuticas.

- Identificar los distintos instrumentos que se utilizan para evaluar la calidad de vida del paciente pediátrico.

- Conocer la percepción de "calidad de vida" por el PPERC

- Conocer la percepción de "calidad de vida" por la familia del PPER.

- Determinar diferencias entre la "calidad de vida" percibida por el PPERC y la "calidad de vida" percibida por la familia. 


\section{Material y métodos}

El diseño empleado es el de un estudio de revisión bibliográfica. Para ello, se hizo una búsqueda exhaustiva en diversas bases de datos con el objetivo de recopilar la mayor producción posible disponible con respecto al tema a tratar en un período comprendido entre 2007-2012.

Las bases de datos empleadas fueron: PubMed, Scopus, Science Direct, ProQuest, Web of Knowledge y SciVerse. El período de consulta de las bases de datos tuvo lugar desde el 20 de Abril de 2012 hasta el 27 de Abril de 2012.

El método de búsqueda consistió en utilizar como palabras clave en las diversas bases de datos: calidad de vida (quality of life); niño (child)/ pediátrico (pediatric)/ adolescent (adolescente); y enfermedad renal crónica (chronic kidney disease)/ enfermedad renal crónica terminal (end-stage renal disease). Empleando los distintos descriptores en singular, plural y que los descriptores empleados estuvieran presentes en el resumen, título del artículo y, si la base de datos lo presenta, en palabras clave.

\section{Los criterios de inclusión fueron:}

- Para ser elegibles los estudios debían tener un diseño de corte transversal ya que el objetivo del estudio era conocer la calidad de vida de PPERC y sus familiares en un momento dado, sin importar por cuánto tiempo mantendrán esta característica ni tampoco cuando la adquirieron. Estos estudios han de tener como resultado principal conocer la calidad de vida del paciente pediátrico con enfermedad renal crónica, ya sea exclusivo o en asociación con otras variables.

- Individuos pediátricos con edades comprendidas desde el nacimiento hasta los 18 años, de cualquier sexo, sanos o enfermos y en diversos casos, familiares/parientes del individuo pediátrico.

- Estudios cuya intervención fuera un cuestionario para determinar la calidad de vida del paciente pediátrico con enfermedad renal crónica.

- Un período de tiempo de 5 años desde el momento de la búsqueda o los artículos publicados desde el 01/01/2007 hasta la actualidad, en función de la base de datos consultada. Con el objetivo de obtener información lo más actualizada posible.

\section{Los criterios de exclusión fueron:}

- Al inicio de la búsqueda, se aceptaron artículos científicos y revisiones para obtener mayor conoci- miento del tema a tratar. Pero posteriormente, sólo se aceptarán los artículos científicos.

- Sólo se aceptaron artículos escritos en inglés o en castellano, puesto que son las dos lenguas con mayor producción científica y que el revisor conoce.

\section{Selección de artículos}

Para iniciar la búsqueda no se incluyeron los criterios de inclusión/exclusión en las diversas bases de datos para ver la cantidad de producción científica existente en el tema y obtener mayor conocimiento en el tema. Posteriormente, si se colocaron los criterios de inclusión/exclusión en las bases de datos.

Diversas bases de datos no permitían introducir como criterios de exclusión el lenguaje del artículo ni permitía sólo obtener artículos científicos (no excluía las revisiones, cartas al director,...). Una vez obtenida la producción científica empleando los descriptores en las bases de datos con los distintos métodos de búsqueda, los artículos extraídos se recopilaron en una carpeta.

Posteriormente se procedió a la lectura de los resúmenes y títulos de todos los artículos, teniendo en cuenta los criterios de inclusión y exclusión, con el objetivo de eliminar los que no tuvieran relación con el tema tratado y eliminar los que estuviesen repetidos. En este punto se eliminaron los artículos de revisión, cartas al director,... artículos que no se encontraban en inglés o castellano, puesto que las demás lenguas de las que existe producción sobre el tema, el investigador al no tener dominio de la lengua las excluyó.

Tras este proceso de cribado, se procedió a recopilar toda la producción científica y eliminar aquellos artículos que pudieran estar repetidos y se volvió a hacer una nueva lectura de los artículos que anteriormente habían pasado el cribado pero que se dudaba de su relación con el tema a tratar, revisando el artículo completo.

Finalmente, los artículos recopilados seleccionados se almacenaron en un mismo archivo o carpeta, exceptuando aquellos a los que no se ha podido acceder. En ese momento, los artículos se analizaron a través de una tabla en la que se determinaba el tipo de cuestionario empleado (específico y/o genérico), la muestra, la modalidad de tratamiento, la calidad metodológica del artículo, si presenta cuestionario para los padres y el tipo de estudio. 
Dichos artículos seleccionados se dividieron, a su vez, en dos categorías: aquellos cuestionarios genéricos, independientes del estado de salud-enfermedad y que analizan contenidos comunes a cualquier situación e intentan dar una aproximación general del estado de salud; $y$ aquellos cuestionarios específicos, dirigidos a una enfermedad y que sus dimensiones intentan profundizar en aspectos de la calidad de vida de la enfermedad renal crónica en el paciente pediátrico, capturando con mayor precisión el impacto de los tratamientos y de las intervenciones sanitarias.

\section{Evaluación crítica de los informes seleccionados}

Se realizó una evaluación de la calidad de los artículos finalmente seleccionados. Para analizar la validez de un estudio de corte transversal, se dispone en la literatura de alguna guía que se basa en aquellos aspectos metodológicos. La herramienta utilizada fue una lista-declaración denominada STROBE versión 4 adaptada a estudios de corte transversal publicada en Octubre/Noviembre de 2007.

\section{Resultados}

\section{Selección de estudios preliminar}

En función de las distintas bases de datos el método de búsqueda varió, empleando distintos métodos. Los artículos localizados se encuentran en la Tabla 1.

En total, de los 79 documentos preseleccionados de todas las bases de datos, tras eliminar los repetidos se quedaran 28 que cumplían con los criterios de inclusión/exclusión establecidos en este estudio de revisión. (Tabla 2).

\section{Análisis de los resultados}

De los 28 documentos seleccionados; 15 se eliminaron tras la lectura del texto completo por los motivos expuestos en la Tabla 3.

\begin{tabular}{|l|c|c|c|c|c|c|c|}
\hline Base de datos & PubMed & Proquest & Scopus & $\begin{array}{c}\text { Science } \\
\text { Direct }\end{array}$ & SciVerse & $\begin{array}{c}\text { Web of } \\
\text { Knowledge }\end{array}$ & $\begin{array}{c}\text { Total de } \\
\text { publicaciones }\end{array}$ \\
\hline $\begin{array}{l}\text { Total de publicaciones } \\
\text { localizadas }\end{array}$ & 46 & 4 & 329 & 17 & 404 & 27 & 827 \\
\hline $\begin{array}{l}\text { Total de publicaciones } \\
\text { preseleccionadas }\end{array}$ & 11 & 1 & 21 & 7 & 26 & 13 & 79 \\
\hline
\end{tabular}

Tabla 1. Número de publicaciones localizadas en cada base de datos (selección preliminar)

\begin{tabular}{|l|c|c|c|c|c|c|c|}
\hline $\begin{array}{l}\text { Total de publicaciones } \\
\text { preseleccionadas }\end{array}$ & $\begin{array}{c}\text { Artículos } \\
\text { repetidos }\end{array}$ & Revisiones & $\begin{array}{c}\text { Presentación a } \\
\text { congreso }\end{array}$ & $\begin{array}{c}\text { Informe } \\
\text { breve }\end{array}$ & Intervención & Diseño \\
\hline 79 & 51 & 3 & 2 & 1 & 3 & 2 \\
\hline
\end{tabular}

Tabla 2. Número de artículos excluidos de la selección según el criterio incumplido (primera selección)

\begin{tabular}{|l|l|}
\hline Artículos excluidos & Causa de la exclusión \\
\hline $\begin{array}{l}\text { Goldstein, SL. et al., 2007; Goldstein, SL. et al., 2009; Marciano, } \\
\text { RC. et al., 2010. }\end{array}$ & Se trata de revisiones \\
\hline $\begin{array}{l}\text { Tromp, M. et al., 2010; Rosenkranz, J. et al., 2007. } \\
\text { Van Bergen, M. et al., 2009 }\end{array}$ & Se trata de presentaciones a congreso \\
\hline Aparicio López C. et al., 2010; Goldstein SL. et al., 2008 & Es un informe breve \\
\hline Dodson JL. et al., 2009; Dodson JL. et al., 2008 & $\begin{array}{l}\text { No determina la calidad de vida en ninguna modalidad } \\
\text { terapéutica de ERC }\end{array}$ \\
\hline Van Bergen M. et al., 2009 & Se trata de un estudio prospectivo, observacional \\
\hline Amr M. et al., 2007 & No se trata de un estudio transversal \\
\hline Riaño-Galan I. et al., 2009; Diseth TH. et al., 2011; Tay LS. et al., 2011 & El resumen no proporciona suficientes datos como para incluirlo \\
\hline
\end{tabular}

Tabla 3. Artículos excluidos tras la lectura del texto completo 
[ P. Jesús López Soto ]

Calidad de vida en el paciente pediátrico con Enfermedad Renal Crónica

\begin{tabular}{|c|c|c|c|c|c|c|}
\hline & Tipo de cuestionario & $\begin{array}{l}\text { Muestra ( } n \% \\
\text { edad en años) }\end{array}$ & Modalidad de tto $\left(n^{\circ}\right)$ & $\begin{array}{l}\text { Informe } \\
\text { parental }\end{array}$ & $\begin{array}{l}\text { Tipo de } \\
\text { estudio }\end{array}$ & 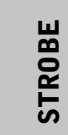 \\
\hline 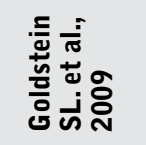 & $\begin{array}{l}\text { Genérico y específico } \\
\text { (PedsQL 4.0, PedsQL } \\
\text { ESRD y PedsQL Family } \\
\text { Impact Module) }\end{array}$ & $\begin{array}{l}8 / 2-4 \\
19 / 5-7 \\
48 / 8-12 \\
125 / 13-18\end{array}$ & $\begin{array}{l}\text { HD (30); DP (45); trasplantados } \\
\text { (125) }\end{array}$ & $\mathrm{Si}$ & Transversal & 17 \\
\hline 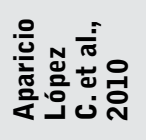 & $\begin{array}{l}\text { Específico } \\
\text { (TECAVNER) }\end{array}$ & $\begin{array}{l}42 />9 \\
29 /<9\end{array}$ & $\begin{array}{l}\text { Tratamiento conservador (22); } \\
\text { trasplantados (33); terapia } \\
\text { sustitutiva (16): DP (11) y } \\
\text { HD(5) }\end{array}$ & $\mathrm{Si}$ & Transversal & 18 \\
\hline 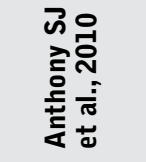 & $\begin{array}{l}\text { Genérico y específico } \\
\text { (PedsQL 4.0, PedsQL } \\
\text { ESRD, VAQOL y PedsQL } \\
\text { Family Impact Module) }\end{array}$ & $\begin{array}{l}12 / 13-18 \\
3 / 8-12 \\
6 / 2-7\end{array}$ & Trasplantados & $\mathrm{Si}$ & Transversal & 19 \\
\hline 总 & $\begin{array}{l}\text { Genérico } \\
\text { (CHIP-AE) }\end{array}$ & 113/10-18 & & No & Transversal & 17 \\
\hline 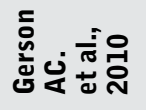 & $\begin{array}{l}\text { Genérico } \\
\text { (PedsQL 4.0) }\end{array}$ & $402 / 2-16$ & & $\mathrm{Si}$ & Transversal & 20 \\
\hline 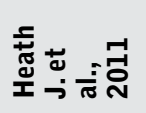 & $\begin{array}{l}\text { Genérico } \\
\text { (GCQ) }\end{array}$ & $\begin{array}{l}124 / 6-14 \\
101 / 14-18\end{array}$ & $\begin{array}{l}\text { Terapia sustitutiva (47): HD } \\
\text { (23) y DP (24); trasplantes } \\
\text { (128) y tto conservador (49) }\end{array}$ & No & $\begin{array}{l}\text { Transversal y } \\
\text { longitudinal }\end{array}$ & 19 \\
\hline 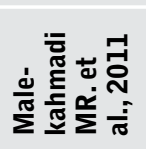 & $\begin{array}{l}\text { Genérico } \\
\text { (SF-36) }\end{array}$ & $55 /<18$ & Trasplantados & No & Transversal & 17 \\
\hline 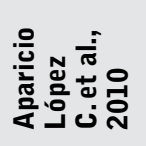 & $\begin{array}{l}\text { Genérico } \\
\text { (M0S-SF-20) }\end{array}$ & $\begin{array}{l}71 / 12.8 \pm 6.4 \\
57 / 10.5 \pm 6.2\end{array}$ & $\begin{array}{l}\text { Tratamiento conservador (22); } \\
\text { trasplantados (33) y terapia } \\
\text { sustitutiva (16): DP (11) y HD } \\
\text { (5) }\end{array}$ & Si & Transversal & 17 \\
\hline 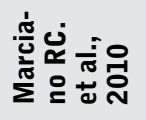 & $\begin{array}{l}\text { Genérico } \\
\text { (PedsQL 4.0) }\end{array}$ & $136 / 2-18$ & $\begin{array}{l}\text { Tratamiento conservador (75); } \\
\text { trasplantados (22) y terapia } \\
\text { sustitutiva (39) }\end{array}$ & $\mathrm{Si}$ & Transversal & 21 \\
\hline 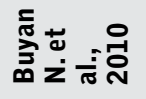 & $\begin{array}{l}\text { Genérico } \\
\text { (KINDL) }\end{array}$ & $\begin{array}{l}211 / 4-18 \\
232 / 4-18\end{array}$ & Trasplantados (139); DP (72) & $\mathrm{Si}$ & Transversal & 18 \\
\hline 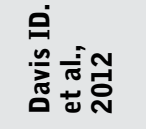 & $\begin{array}{l}\text { Genérico } \\
\text { (PedsQL 4.0) }\end{array}$ & 159/5-18 & $\begin{array}{l}\text { Tratamiento conservador (68); } \\
\text { trasplantados (61) y terapia } \\
\text { sustitutiva (30) }\end{array}$ & $\mathrm{Si}$ & Transversal & 20 \\
\hline 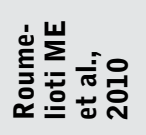 & $\begin{array}{l}\text { Genérico } \\
\text { (PedsQL 4.0) }\end{array}$ & 301/10.8-16.2 & & $\mathrm{Si}$ & $\begin{array}{l}\text { Transversal } \\
\text { dentro de un } \\
\text { estudio de } \\
\text { cohortes }\end{array}$ & 21 \\
\hline 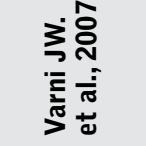 & $\begin{array}{l}\text { Genérico } \\
\text { (PedsQL 4.0) }\end{array}$ & $\begin{array}{l}96 / 8-18 \\
2404 \text { otras } \\
\text { enfermedades }\end{array}$ & $\begin{array}{l}\text { Trasplantados (45); HD (32) y } \\
\text { PD (19) }\end{array}$ & $\mathrm{Si}$ & Transversal & 20 \\
\hline
\end{tabular}

Tabla 4. Calidad de los estudios seleccionados según STROBE. Clasificación por modalidad, corte de estudio, muestra, tipo de cuestionario e informe parental 
Los 13 documentos restantes son artículos completos. Estos 13 documentos se clasificaron en función del tipo de cuestionario empleado (específico y/o genérico), la muestra (número y edad), la modalidad de tratamiento, si presenta cuestionario a los padres y el tipo de estudio. (Tabla 4)

Del análisis de los informes se extrajeron dos grupos según el tipo de cuestionario: 10 que empleaban cuestionarios generales, 1 artículo que empleó un cuestionario específico para determinar la calidad de vida del paciente pediátrico con ERC y 2 que empleaban tanto genérico como específico.

\section{Descripción de los resultados}

En los artículos encontrados, se detecta que los cuestionarios generales sobre calidad de vida son más empleados que los específicos. En los cuestionarios generales, se distinguen dos variables de estudio principales: la modalidad terapéutica que determina la calidad de vida del paciente con ERC comparándola con las distintas modalidades, un grupo control u otra enfermedad; $y$ determinados factores que determinan la calidad de vida del paciente con ERC, comparándolos con otra modalidad terapéutica o dentro de la misma modalidad.

En el cuestionario específico, la variable de estudio más empleada es la modalidad terapéutica, comparando la influencia de la modalidad sobre la calidad de vida de paciente pediátrico con ERC.

Existen dos estudios que emplean ambos tipos de cuestionarios, la variable de estudio más estudiada es la modalidad terapéutica y además compara los resultados obtenidos por un cuestionario y por otro.

Cabe resaltar que la mayor parte de los estudios emplean un informe parental para determinar la calidad de vida del paciente pediátrico, desde el punto de vista de la familia/parientes próximos.

Además la mayor parte los estudios emplean el cuestionario genérico PedsQL 4.0 Generic Core Scales.

Dentro de cada tipo de cuestionario se encuentran diversas variables que van a influir en la calidad de vida del paciente pediátrico, como puede ser problemas de sueño ${ }^{14,15}$, desórdenes emocionales ${ }^{16}$, ingresos familiares ${ }^{17}$, etc.

\section{Cuestionarios generales}

\section{- Modalidad terapéutica}

Se evaluó con abordajes muy diferentes. De los diez estudios que emplean cuestionarios generales, en siete de ellos $11,12,14,16,17,18,19$ se evaluó la influencia de las distintas modalidades terapéuticas (tratamiento sustitutivo, trasplantados, tratamiento conservador) en la calidad de vida.

En un estudio que utiliza un cuestionario general, empleó la variable "trasplantado" para determinar la calidad de vida de los pacientes pediátricos solamente sometidos a esa modalidad ${ }^{17}$.

Los resultados respecto a los diferentes tipos de modalidad terapéutica mostraron que la calidad de vida del paciente que ha sido trasplantado presentaban mejoras en dominios de los distintos cuestionarios que la de las demás modalidades terapéuticas (hemodiálisis y diálisis peritoneal) y que con respecto a la población general existen discrepancias sobre si poseen la misma calidad de vida o inferior. Solo en dos artículos se mostraron diferencias significativas entre trasplantados y las demás modalidades terapéuticas ${ }^{12,14}$.

Al comparar entre modalidad de tratamiento sustitutivo (HD y DP) no se encontraron diferencias estadísticamente significativas, aunque se encuentran una ligera mejora en la calidad de vida de los pacientes con DP con respecto a los pacientes sometidos a $\mathrm{HD}^{12}$.

\section{- Percepción de la familia/cuidadores}

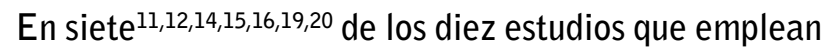
cuestionarios generales, además de la percepción del paciente de su propia salud, la familia también es evaluada para comprobar su percepción. De esta forma se evalúan los dos puntos de vista y se determinan las discrepancias que pudieran existir.

En la mayoría de los estudios (6 de 7)9,14,15,16,19,21 las puntuaciones de calidad de vida general obtenidas por los cuestionarios Ilevados a cabo por los familiares son peores que las percepciones del propio paciente. Consideran que el paciente goza de menor calidad de vida que lo que ellos perciben. 
Aunque en un estudio las puntuaciones de calidad de vida general son mejores en familiares que las relatadas por el propio paciente ${ }^{12}$. En ambos casos las diferencias no son significativas, aunque si lo sean en factores que inciden sobre la calidad de vida general.

En todos los estudios se ha determinado que existe una leve correlación con lo relatado por el niño. Además, en diversos casos es primordial (enfermos mentales, poca edad,...) para determinar la calidad de vida del paciente pediátrico ${ }^{22}$.

\section{- Problemas de sueño}

Este resultado fue encontrado en dos de los diez artículos $^{14,15}$ que emplean cuestionarios generales. En los dos se obtuvo significación estadística que asociaba problemas de sueño con bajas puntuaciones de calidad de vida.

Los problemas de sueño además de correlacionarse con puntuaciones bajas de calidad de su vida, a su vez, se ven influenciados por factores como modalidad terapéutica, tasa de filtrado glomerular (GFR) y factores socioeconómicos.

\section{- Factores psicológicos}

En dos artículos ${ }^{16,17}$ de los diez que emplean cuestionarios generales, se ha determinado este tema. En Malekahmadi MR, et al. (2011) se vio el gran poder predictivo relativo en pacientes post-trasplantados, determinando que es más importante que los factores socioeconómicos y clínicos. En Marciano RC, et al. (2011), se demostró significativamente que la prevalencia de desórdenes de comportamiento y emocionales se correlacionaba con una pérdida de la calidad de vida con respecto a la población normal.

\section{- Tasa de filtrado glomerular (GFR)}

En los diez estudios en los que se determinaba la calidad de vida con cuestionarios generales, en tres de ellos 914,15 se relacionó la GFR con la calidad de vida. El abordaje de esta variable fue muy heterogéneo. En dos de ellos ${ }^{14,15}$ se determinó la relación de la GFR sobre la calidad de vida indirectamente, a través de problemas de sueño, que se vio que correlacionaba prevalencia de problemas de sueño con calidad de vida. En Roumelioti
$M E$, et al. (2010) se mostró significación estadística de que la GFR determina los problemas de sueño y consigo la calidad de vida.

El otro abordaje 9 fue más simple, se relaciono GFR con calidad de vida pero no se encontraron diferencias significativas, solo una puntuación ligeramente mejor cuanto mayor GFR exista.

\section{- Factores socioeconómicos}

Los diferentes factores socioeconómicos que se incluyen en los estudios que emplean cuestionarios generales van desde el género, edad, ingresos familiares, religión hasta incluso educación maternal. En un estudio ${ }^{17}$ se determina que los factores socioeconómicos junto con los factores psicológicos y clínicos constituyen el $70 \%$ de la variación en la calidad del paciente pediátrico trasplantado.

En otro estudio ${ }^{16}$ se asociaron significativamente baja calidad de vida con pacientes con edades $<10$ años y ateos. En dos estudios ${ }^{15,17}$ se asociaron significativamente bajos ingresos familiar mensual con peor calidad de vida. E incluso, en un estudio se asoció peor educación maternal con peor calidad de vida.

\section{- Causa de la ERC}

Fue estudiado en dos estudios. ${ }^{9,23}$. En Dodson JL, et al. (2007) se hacía referencia a la ausencia de diferencias estadísticamente significativas entre los pacientes pediátricos con ERC debido a enfermedades congénitas urológicas y pacientes con ERC debido a otros factores. $Y$ en Gerson AC, et al. (2010) no se aportaban datos al respecto pero consideraba a la causa de la ERC como variable clínica que determina la calidad de vida del paciente pediátrico sometido a trasplante.

\section{Cuestionarios específicos - Modalidad terapéutica}

El estudion ${ }^{11}$ que emplea solamente el cuestionario específico determinó la relación de la variable "modalidad terapéutica" con la calidad de vida. En este estudio, al comparar entre modalidad de tratamiento sustitutivo (HD y DP) no se encontraron diferencias estadísticamente significativas en la calidad de vida general, aunque si en diversos dominios del cuestionario. Aunque no exista diferencias significativas se encuentran una ligera me- 
jora en la calidad de vida de los pacientes con DP con respecto a los pacientes sometidos a HD. En este estudio, los padres consideran que la calidad de vida es peor que la percibida por sus hijos. Los padres infravaloran la calidad de vida de sus hijos. No hay concordancia entre padres e hijos menores de 12 años, pero va mejorando conforme aumenta la edad.

\section{Cuestionarios generales y específicos - Modalidad terapéutica}

En uno de los dos estudios ${ }^{22,24}$ que emplean tanto cuestionarios específicos como generales se determinó la modalidad terapéutica como variable de la calidad de vida, no obteniendo diferencias significativas entre los pacientes pediátricos trasplantados y los que se encuentran en tratamiento sustitutivo. Solo se encontró una ligera mejora en la calidad de vida.

\section{- Percepción del familiar}

En los dos estudios ${ }^{22,24}$ la percepción del familiar difiere a la percepción del niño. Existen discordancias entre lo informado por el padre y el hijo. La actitud del padre siempre tiende a sobreproteger al hijo. La correlación entre lo informado por el hijo y el familiar, va a ir aumentando con el paso de los años.

\section{Discusión}

El hallazgo principal de esta revisión es la multidimensionalidad de la calidad de vida en el paciente pediátrico con enfermedad renal crónica y la cantidad de variables de resultado obtenidas que inciden sobre el concepto "calidad de vida", desde la causa de la enfermedad hasta el nivel de educación maternal.

Este carácter multidimensional es el que más problemas conlleva a la hora de determinar la calidad de vida en toda persona, puesto que no se ha dado todavía una respuesta clara de los parámetros 0 áreas que participan en la vida de la persona ni el peso que hay que darle a cada una de esas áreas en el producto final de la calidad de vida4.

En la revisión la calidad de vida se han observado relaciones con variables clínicas, psicológicas y socioeco- nómicas, pero aun así, no se tiene una respuesta clara de las variables que determinan la calidad de vida.

Además se ha visto el carácter subjetivo que tiene, Jirojanakul et al. (2003) sugieren que los factores de salud "per $s e^{\prime \prime}$ no impactan significativamente sobre la $\mathrm{CV}$, ya que es determinada por factores tales como las circunstancias personales relativas a las expectativas..$^{25}$ De ahí, que se aborde también el carácter psicosocial de la CV. ${ }^{18}$

Esta revisión también refleja que existe una variada disponibilidad de cuestionarios genéricos, pero no tan variada de cuestionarios específicos para valorar la calidad de vida con esta enfermedad. También, la mayoría de los estudios se limitan a adolescentes.

Se aprecia que la consideración general de que los niños no han sido capaces de responder sobre su propia CV ha ido cambiando ${ }^{11}$. En los últimos 5 años, cada vez hay más estudios basados en la percepción del niño. Se ha visto que la percepción del paciente pediátrico y de sus padres no ha de ser la misma, por eso ha de tenerse en cuenta cómo se siente el paciente pediátrico dicha enfermedad y el tratamiento. Por tanto, sería aconsejable que toda evaluación actual de la CV en paciente pediátrico contara con su opinión ${ }^{11,21}$.

La sensibilización sobre cómo se siente el paciente pediátrico en el tratamiento es el tema más estudiado en la revisión. Algunos estudios ${ }^{11,26}$ afirman que los estudios con cuestionarios generales pueden no discriminar diferencias entre diferentes tratamientos al tratarse de cuestionarios generales no específicos para la enfermedad renal.

El cuestionario genérico si puede permitir comparar a pacientes pediátricos sanos con ERC y si se obtienen diferencias esperadas como que los sanos poseen mejor CV que los pacientes con ERC ${ }^{16,21}$. En los cuestionarios específicos se encuentran diferencias según la modalidad terapéutica ${ }^{11,22}$, que aunque se encuentran en algunos cuestionarios generales ${ }^{12,21}$, no están destinados para tal fin.

Se ha visto la calidad de vida de los pacientes con ERC comparada con otras 10 enfermedades crónicas ${ }^{19}$, 
reportando el paciente pediátrico con ERC peor CV que el grupo sano y que el diabético, y no significativamente peor que los pacientes pediátricos con problemas gastrointestinales, cardíacos y asmáticos. Siendo significativamente mejor que el grupo con parálisis cerebral y no significativamente mejor que enfermos mentales, cáncer y reumáticos. Por tanto, se ha visto que dentro de las enfermedades crónicas, la ERC en niños posee una baja CV.

Debido a la percepción que se tiene de cómo se siente el paciente, en lugar de como creen los médicos que debería sentirse en función de las medidas clínicas. La terapia debe ser evaluada si es más o menos probable conseguir una vida digna de ser vivida, tanto en términos sociales y psicológicos como en términos físicos ${ }^{27}$. Por ello, otro principal hallazgo de la revisión es la determinación de los trasplantes como modalidad terapéutica con mayor CV después del tratamiento conservador. El trasplante también representa la forma preferida de terapia de remplazamiento renal desde una perspectiva médica, pues los pacientes pediátricos demuestran mejor que aquellos que reciben diálisis ${ }^{28}$. La mejor CVRS del trasplante con respecto al tratamiento sustitutivo, lo convierte en la modalidad terapéutica de elección. En la revisión, se han obtenido mejores puntuaciones dentro del tratamiento sustitutivo, en la diálisis peritoneal que hemodiálisis, que aunque no se han obtenido diferencias significativas existe una ligera mejora ${ }^{11,12,22}$.

Otro hallazgo es la percepción proteccionista de los familiares, con respecto a lo percibido por el propio paciente pediátrico, que aunque existe una leve correlación, su percepción no equivale a lo percibido por el paciente pediátrico, ya que, siempre tienden a valorar peor los distintos dominios de los cuestionarios ${ }^{12,24}$.

También se observa que cuando aumenta la edad del paciente pediátrico, la percepción del paciente pediátrico se va correlacionando más con lo percibido por el familiar/cuidador ${ }^{11}$.

Otro hallazgo fue la importancia de las variables psicológicas $^{16}$ y socioeconómica ${ }^{17}$. Como factores socioeconómicos adversos (bajos ingresos familiares e incluso, baja educación maternal $)^{20}$ determinan una peor $\mathrm{CV}$ en estos pacien- tes, y como se relacionan la prevalencia de desórdenes emocionales y de comportamiento se correlacionan con la CV. También como la causa de la ERC puede determinar la $\mathrm{CV}^{23}$ e incluso, el GFR puede determinarla ${ }^{20}$.

Para finalizar, en la revisión también aparecen la prevalencia de problemas de sueño asociados a la CV de los pacientes pediátrico con ERC ${ }^{14,15}$. Y como el GFR también puede determinar la presencia de problemas de sueño, y consigo, la calidad de vida.

\section{Conclusiones}

1. Se ha encontrado que los pacientes pediátricos con ERC tienen una CV más baja con respecto a la CV de la población general y otras enfermedades crónicas como la diabetes.

2. Los niños sometidos a tratamiento conservador y trasplantes poseen mejor calidad de vida que los sometidos a tratamiento sustitutivo (DP y HD)

3. No se han encontrado diferencias significativas en la CV entre pacientes pediátricos con ERC sometidos a DP y a HD.

4. Existe una leve correlación entre lo informado por el familiar/cuidador y lo informado por el paciente pediátrico con ERC, en cuanto a la CV.

5. Los cuestionarios específicos a la enfermedad dan mayores diferencias y permiten hacer distinciones entre las distintas modalidades terapéuticas.

6. Las variables psicológicas y socioeconómicas, no solo las clínicas, pueden determinar la CV del paciente con ERC.

\section{Agradecimientos}

Es obligado por mi parte agradecer a la Universidad de Córdoba, y en especial al programa de Doctorado en Biomedicina por hacer posible el desarrollo de este trabajo.
Recibido: 7 septiembre 2012
Revisado: 30 septiembre 2012
Modificado: 19 noviembre 2012
Aceptado: 20 noviembre 2012 


\section{Bibliografía}

1. National Kidney Foundation. KDOQI clinical practice guidelines for chronic kidney disease: evaluation, classification, and stratification. Am J Kidney Dis 2002; 39 [2 Suppl 1]:S1-S266.

2. Gill TM, Feinstein AR. A critical appraisal of the quality of life Measurements. JAMA 1994; 272(8): 619-626.

3. Asociación Española de Nefrología Pediátrica. Noticias [en línea]: de la AENP http://www. aenp.es/index.php?option=com_content\&view $=$ article\&id=108: presentacion-del-registro-espanol-de-insuficiencia-renal-cronica-terminalrepir\&catid=13: noticias-aenp\&Itemid=75 [Consulta: 21 de Abril de 2012].

4. Aparicio López, María Cristina. "Calidad de vida en pacientes pediátricos con enfermedad renal crónica y repercusión psicológica en sus padres". Directores: Ángel Pedro Carrillo Álvarez, Angustias Fernández Escribano. Universidad Complutense de Madrid, Facultad de Medicina, Departamento de Pediatría, 2003.

5. Levi R, Drotar D. Critical issues and needs in health-related quality of life assessment of children and adolescents with chronic health condition. In: Drotar D, editor. Measuring health-related quality of life in children and adolescents Implications for research and practice. Hillsdale, New Jersey: Lawrence Erlbaum Associates; 1998. p. 3-24.

6. Schwartzmann L. Calidad de vida relacionada con la salud: aspectos conceptuales. Ciencia y enfermería 2003; 9(2): 9-21.

7. TESTA M. Current Concepts: Assessment of Quality-of-Life Outcomes. N Engl J Med, 1996; 334(13), 835-840.

8. Nanda U, Andresen E. Health related Quality of life, a guide por the health professional. Evaluation $\&$ the Health Professions 1998; 21: 179-215.

9. Gerson A, Hwang W, FIorenza J, Barth K, Kaskel F, Weiss L, Zelikovsky N, Fivush B, Furth S. Anemia and health-related quality of life in adolescents with chronic kidney disease. Am J Kidney Dis 2004; 44:1017-1023.

10. Burke C. Testing an Asthma quality of life model. Jour $\neg$ nal of theory construction \& testing 2001; 5: 38-44.

11. Aparicio C, Fernández A, Izquierdo E, Luque A, Garrido $G$. Medida mediante un test específico de la calidad de vida relacionada con la salud en niños con enfermedad renal crónica. Influencia del tratamiento. Nefrología 2010; 30 (2):177-84.

12. Buyan N, Türkmen MA, Bilge I, Baskin E, Haberal M, Bilginer Y, Mir S, Emre S, Akman s, Ozkaya 0, Fidan K, Alpay H, Kavukcu s, Sever L, Özçakar ZB, Dogrucan N. Quality of life in children with chronic kidney disease (with child and parente assessments). Pediatr Neprhol 2010; 25:14871496.

13. Patrick DL, Deyo RA. Generic and disease-specific measures in assessing health status and quality of life. Med Care. 1989;27(3 Suppl):S217-S232.

14. Davis ID, Greenbaum LA, Gipson D, Wu LL, Sinha $\mathrm{R}$, Matsuda-Abedini M, Emancipator JL, Lane JC, Hodgkins K, Nailescu C, Barletta GM, Arora S, Mahan JD, Rosen CL. Prevalence of sleep disturbances in children and adolescents with chronic kidney disease. Pediatr Nephrol. 2012; 27(3):451-9.

15. Roumelioti ME, Wentz $A$, Schneider MF, Gerson AC, Hooper S, Benfield M, Warady BA, Furth SL, Unruh ML. Sleep and fatigue symptoms in children and adolescents with CKD: a cross-sectional analysis from the chronic kidney disease in children (CKiD) study. Am J Kidney Dis. 2010; 55(2):269-80.

16. Marciano RC, Soares CMB, Diniz JSS, Lima EM, Silva JMP, Canhestro MR, Gazzinelli A, Melo CCD, Dias CS, Simões e Silva AC, Correa H, Oliveira EA. Behavioral disorders and low quality of life in children and adolescents with chronic kidney disease. Pediatr Nephrol 2011; 26:281-290.

17. Malekahmadi MR, Rahimzadeh S, Dezfuli Nejad $M L$, Lankarani MM, Einollahi B, Assari S. Importance of socioeconomic, clinical, and psychological factors on health-related quality of life in adoles- 
cents after kidney transplant. Exp Clin Transplant 2011; 9(1):50-5.

18. Heath J, MacKinlay D, Watson AR, Hames A, Wirz L, Scott S, Klewchuk E, Milford D, McHugh K. Selfreported quality of life in children and young people with chronic kidney disease. Pediatr Nephrol 2011; 26:767-773.

19. Varni JW, Limbers CA, Burwinckle TM. Impaired health-related quality of life in children and adolescents with chronic conditions: a comparative analysis of 10 disease clusters and 33 disease categories/severities utilizing the PedsQLTM 4.0 Generic Core Scales. Health and Quality of Life Outcomes 2007; 5:43.

20. Gerson $A C$, Wentz $A$, Abraham $A B$, Mendley $S R$, Hooper SR, Butler RW, Gipson DS, Lande MB, Shinnar S, Moxey-Mims MM, Warady BA, Furth SL. Health-related quality of life of children with mild to moderate chornic kidney disease. Pediatrics 2010; 125(2):e349-57.

21. Aparicio $C$, Fernández $A$, Izquierdo $E$, Luque $A$, Garrido G. Calidad de vida percibida por niños con enfermedad renal crónica y por sus padres. Nefrología 2010; 30 (1);103-9.

22. Goldstein $S L$, Rosburg NM, Warady BA, Seikaly M, McDonald R, Limbers C, Varni JW. Pediatric end stage renal disease health-related quality of life differs by modality: a PedsQL EsRD analysis. Pediatr Nephrol 2009; 24:1553-1560.
23. Dodson JL, Diener-West M, Gerson AC, Kaskel FJ, Furth SL. An assessment of health related quality of life using the child health and illness profile-adolescent edition in adolescents with chronic kidney disease due to underlying urological disorders. J Urol. 2007; 178(2):660-5.

24. Anthony SJ, Hebert $D$, Todd $L$, Korus $M$, Langlois V, Pool R, Robinson LA, Williams A, Polloch-BarZiv SM. Child and parental perspectives of multidimensional quality of life outcomes after kidney transplantation. Pediatr transplantation 2010; 14:249-256.

25. Jirojanakul P, Skevington SM, Hudson J. Predicting young children's quality of life. Soc Sci Med. 2003; 57:1277-1288.

26. McKenna $A M L$, Keating $E$, Vigneux $A$, Stevens $S$, Williams A, Geary DF. Quality of life in children with chronic kidney disease-patient and caregiver assessments. Neprhrol Dial Transplant 2006; 21:1899-905.

27. Bobes J, Bousoño García M, García-Portilla MP, Pedregal Sánchez J, editores. Calidad de vida y sueño. Novograf, S.L.

28. McDonald SP, Craig JC. Long-term survival of children with end-stage renal disease. $\mathrm{N} \mathrm{ENgl} \mathrm{J} \mathrm{Med}$ 2004; 350:2654-2662. 